Illinois State University

ISU ReD: Research and eData

Theses and Dissertations

$1-21-2018$

\title{
Dietary Sodium And Blood Pressure Changes In Hemodialysis Patients Undergoing A Liberalized Renal Diet Intervention
}

Lauren Eve Chan

Illinois State University, laurenevechan@gmail.com

Follow this and additional works at: https://ir.library.illinoisstate.edu/etd

Part of the Human and Clinical Nutrition Commons, and the Medicine and Health Sciences Commons

\section{Recommended Citation}

Chan, Lauren Eve, "Dietary Sodium And Blood Pressure Changes In Hemodialysis Patients Undergoing A Liberalized Renal Diet Intervention" (2018). Theses and Dissertations. 848.

https://ir.library.illinoisstate.edu/etd/848

This Thesis is brought to you for free and open access by ISU ReD: Research and eData. It has been accepted for inclusion in Theses and Dissertations by an authorized administrator of ISU ReD: Research and eData. For more information, please contact ISUReD@ilstu.edu. 


\title{
DIETARY SODIUM AND BLOOD PRESSURE CHANGES
}

\author{
IN HEMODIALYSIS PATIENTS UNDERGOING A \\ LIBERALIZED RENAL DIET INTERVENTION
}

\author{
Lauren Eve Chan
}

\section{Pages}

Objective: Investigate the impacts of individual liberalized renal diet counseling in conjunction with a volume reduction hemodialysis (HD) protocol.

Design \& Methods: Twenty-three maintenance HD patients (age $=55.7 \pm 13.3 \mathrm{y}, 47.8 \%$ female), consented and completed this pilot intervention. Across the six-month intervention, participants received thrice weekly dietary counseling about a liberalized renal diet. Liberalized renal dietary guidelines promoted a low sodium diet with greater unprocessed food consumption, decreasing foods eaten outside the home, and increased food label reading. Participant HD sessions were conducted per a volume reduction protocol, gradually decreasing patient post-dialysis weight by removing an additional 200-300 mL/session. Preliminary outcome measures included dietary intake and knowledge, blood pressure (BP), anti-hypertensive medication use, and volume overload (VO).

Results: From baseline (BL) to six months $(6 \mathrm{~m})$, total sodium intake numerically decreased (BL $2886 \pm 1570.6$ vs $6 \mathrm{~m} 2315 \pm 1095 \mathrm{mg}, \mathrm{p}=0.13)$, systolic BP (BL $160 \pm 25$ vs. $6 \mathrm{~m} 156 \pm 23$ $\mathrm{mmHg}, \mathrm{p}=0.56)$ and diastolic $\mathrm{BP}(\mathrm{BL} 81 \pm 20$ vs $6 \mathrm{~m} 79 \pm 15 \mathrm{mmHg}, \mathrm{p}=0.73)$ showed no significant changes, but total number of anti-hypertensive medications prescribed to patients (BL $3 \pm 1$ vs $6 \mathrm{~m} 2 \pm 1$ medications) were significantly reduced ( $\mathrm{p}=0.003)$. Additionally, significant improvements were noted in $\mathrm{VO}(\mathrm{BL} 3.6 \mathrm{~L} \pm 3.9 \mathrm{~L}$ vs $6 \mathrm{~m} 2.5 \mathrm{~L} \pm 3.5 \mathrm{~L}, \mathrm{p}=0.01)$. 
Conclusion: Liberalized renal diet education had little effect on sodium intake, likely contributing only minimally to BP control. Volume reduction protocol with gradual reduction of post dialysis weight resulted in significantly decreased $\mathrm{VO}$, and maintenance of $\mathrm{BP}$ with coinciding decreases in anti-hypertensive medication usage. Our findings document intervention opportunities to improve BP and decrease medication usage for HD patients.

KEYWORDS: hemodialysis, liberalized renal diet, volume reduction, blood pressure, sodium reduction, anti-hypertensive medication 


\section{DIETARY SODIUM AND BLOOD PRESSURE CHANGES \\ IN HEMODIALYSIS PATIENTS UNDERGOING A

\author{
LIBERALIZED RENAL DIET INTERVENTION
}

LAUREN EVE CHAN

A Thesis Submitted in Partial Fulfillment of the Requirements for the Degree of

MASTER OF SCIENCE

Department of Family and Consumer Sciences

ILLINOIS STATE UNIVERSITY 
Copyright 2018 Lauren Eve Chan 


\title{
DIETARY SODIUM AND BLOOD PRESSURE CHANGES \\ IN HEMODIALYSIS PATIENTS UNDERGOING A \\ LIBERALIZED RENAL DIET INTERVENTION
}

\author{
LAUREN EVE CHAN
}

COMMITTEE MEMBERS:

Jennifer Barnes, Chair

Ken Wilund

Sally Arnett-Hartwick 


\section{ACKNOWLEDGMENTS}

I would first like to thank my thesis advisor, Dr. Jen Barnes of the Family and Consumer Sciences Department at Illinois State University. Dr. Barnes was an encouraging advisor who significantly influenced my learning and urged me to pursue my own unique, independent research. I am incredibly grateful for Dr. Barnes for her guidance during this process and throughout my entire experience at ISU.

I want to thank Dr. Ken Wilund of the Kinesiology and Community Health Department at the University of Illinois Urbana Champaign, and the members of his lab who assisted in this pilot project. ISU's collaborative relationship with the University of Illinois Urbana-Champaign allows opportunities for meaningful research of this caliber. Without the passionate participation and input of all the lab members from both Illinois State University and University of Illinois Urbana-Champaign, this research could not have been successfully conducted.

I would also like to acknowledge Dr. Sally Arnett-Hartwick of the Illinois State University Department of Family and Consumer Sciences as the second reader of this thesis, and I am gratefully indebted to her for her valuable comments on this thesis.

Finally, I must express my very profound gratitude to my family for providing me with unfailing support and continuous encouragement throughout my years of study and through the process of researching and writing this thesis. This accomplishment would not have been possible without them. Thank you.

L. C. 


\section{CONTENTS}

Page

ACKNOWLEDGMENTS

CONTENTS

TABLES

FIGURES

CHAPTER I: MANUSCRIPT 1

$\begin{array}{ll}\text { Introduction } & 1\end{array}$

$\begin{array}{ll}\text { Methodology } & 4\end{array}$

Design and Subjects $\quad 4$

$\begin{array}{ll}\text { Intervention } & 7\end{array}$

$\begin{array}{ll}\text { Statistical Analysis } & 8\end{array}$

$\begin{array}{ll}\text { Results } & 9\end{array}$

$\begin{array}{ll}\text { Participant Demographics } & 9\end{array}$

Body Fluid and Volume Overload 9

$\begin{array}{ll}\text { Weight } & 9\end{array}$

$\begin{array}{ll}\text { Blood Pressure and Medication Use } & 10\end{array}$

Post Intervention Dietary Intake and Knowledge 10

$\begin{array}{ll}\text { Discussion } & 11\end{array}$

$\begin{array}{ll}\text { Conclusion } & 17\end{array}$

CHAPTER II: LITERATURE REVIEW 18

$\begin{array}{ll}\text { Introduction } & 18\end{array}$

$\begin{array}{ll}\text { Literature Review } & 19\end{array}$ 
The Traditional Renal Diet $\quad 19$

Dietary Sodium 19

$\begin{array}{ll}\text { Dietary Potassium } & 21\end{array}$

$\begin{array}{ll}\text { Dietary Phosphorus } & 22\end{array}$

Traditional Renal Diet Challenges 23

$\begin{array}{ll}\text { Anti-Hypertensive Medications } & 26\end{array}$

$\begin{array}{ll}\text { Ultrafiltration Techniques } & 27\end{array}$

Comparing Hypertension Management Techniques 28

Dietary Sodium Reduction Challenges $\quad 31$

Reconsidering HTN Treatment in the US 32

$\begin{array}{ll}\text { REFERENCES } & 34\end{array}$

APPENDIX A: 24-HOUR RECALL FORM 49

APPENDIX B: PATIENT SUMMARY SHEET $\quad 50$

APPENDIX C: GOALS TEMPLATE 


\section{TABLES}

Table

Page

1. Baseline Patient Characteristics

2. Body Fluid Levels 43

3. Blood Pressure and Medication Outcomes 44

4. Dietary Intake Evaluation 45

5. Dietary Knowledge Evaluation 46 


\section{FIGURES}

Figure $\quad$ Page

1. Reduction in Liters of Volume Overload Following Intervention 47

2. Significant Post-Intervention Improvement in Volume Overload Percentage 48 


\section{CHAPTER I: MANUSCRIPT \\ INTRODUCTION}

Prevalence of end stage renal disease (ESRD) is increasing in the United States (US), with approximately 110,000 new cases each year. ${ }^{1}$ Patients with ESRD typically have less than $15 \%$ function of their kidneys, a glomerular filtration rate (GFR) of $<15 .{ }^{2}$ ESRD patients require either peritoneal dialysis or hemodialysis (HD) or other blood filtration treatments to supplement fluid and metabolic waste removal, with HD being the most common selection. ${ }^{2}$

HD treatments are particularly important to remove minerals such as sodium, potassium, and phosphorus from the blood. These dietary minerals greatly impact fluid balance and can alter blood pressure (BP) levels if their concentrations are irregular. ${ }^{5}$ Increased levels of these minerals, sodium in particular, alter BP when concentrations are greater than normal causing increased fluid retention. ${ }^{6}$ Sodium consumption is also directly tied to thirst, and with additional sodium intake a patient will experience a strong physiological thirst sensation leading them to drink more fluid. ${ }^{6}$ By introducing more fluid to the system, the patient will increase their body fluid volume. ${ }^{6}$ Expanded body fluid, and in turn blood volume, can increase BP, leading many patients to develop or exacerbate pre-existing hypertension (HTN) or other cardiovascular (CV) risks such as aortic stiffness. ${ }^{7}$

Currently, upwards of $80-90 \%$ of HD patients experience HTN. ${ }^{8}$ In an investigation with greater than 30,000 individual HD patients, large gains of body fluid between dialysis sessions, defined as $>4.0 \mathrm{~kg}$, were correlated with higher risk of death over the following two years. ${ }^{9}$ With cardiovascular disease (CVD) being the main cause of death among ESRD patients, encompassing $41 \%$ of patient deaths ${ }^{10} \mathrm{BP}$ management strategies are critical for this population. 
In the US, treatment for HD patients include both lifestyle and pharmaceutical approaches in addition to regular dialysis treatments to maintain healthy $\mathrm{BP} .{ }^{3,4}$

For improved BP and health management, HD patients are often counseled to follow the traditional renal diet to decrease their dietary mineral intake in an effort to manage their interdialytic blood mineral concentrations. Restricted foods include dairy, nuts, whole grains, fruits, and vegetables, making it a complicated and challenging diet to follow. ${ }^{11}$ With recommendations to limit traditionally heart healthy foods, HD patients may be at risk of consuming a pro-atherogenic diet that is low in fiber and micronutrients. ${ }^{11,12}$ In addition, HD patients often experience multiple barriers for dietary change that can vary from struggles learning to utilize new food items ${ }^{13}$, to the desire for more individualized dietary guidance. ${ }^{14}$ Furthermore, most hypertensive American HD patients are prescribed anti-hypertensive medications as the primary treatment to decrease BP. ${ }^{15}$ Although anti-hypertensive BP medications are commonly used, their effectiveness in this population is questionable. For significant BP reduction, some HD patients need upwards of four medications to alter their BP. ${ }^{15,16,17}$ Additionally, medications are expensive to purchase, challenging to monitor and maintain, and can have other unwanted side effects including an increased risk for interdialytic hypotension. ${ }^{5,15,18}$ In efforts to significantly reduce and maintain appropriate BP levels, patients may need alternative treatments to medications.

Controlling dietary sodium intake is an important component to managing BP. Currently recognized as part of the traditional renal diet, limiting sodium intake to less than 2,000 milligrams per day can reduce fluid retention and BP in HD patients. ${ }^{6,19}$ Although sodium recommendations are typically given to HD patients, poor dietary sodium adherence is common. Poor adherence may be related to various barriers for dietary changes, including how confusing 
and overwhelming the traditional renal diet is. ${ }^{11,13,14}$ Recent efforts to simplify the renal diet are being considered to help HD patients focus on sodium reduction to decrease the onset or progression of HTN. Additionally, a more liberalized diet may allow for patients to have greater food selection, more micronutrients, and increased fiber in their diet, a concern for many HD patients. ${ }^{11,20}$

In addition to dietary adaptations, HTN and volume overload (VO) may be altered through volume reduction protocol with the removal of an additional 200-300 mL of fluid per HD session. A patient's dry weight is often an estimation made by a nephrologist, rather than an objective measurement of the patient's edema free body weight, and thus only dialyzing to the dry weight may leave excess fluid on the body and maintain an elevated BP. ${ }^{9,21}$ By seeking to remove a small amount of additional fluid during each dialysis session, the patient may come closer to their actual edema free body weight and gradually reduce their BP. ${ }^{21}$

By reducing post dialysis weight each session and using dietary interventions such as the liberalized renal diet, patients may see a reduction in BP alongside a decreased need for BP medications. In turn, this may result in better CVD outcomes and lower mortality risk for HD patients.

Following a six-month intervention with education on a liberalized renal diet and the use of volume reduction protocol, we hypothesized patients in this pilot study would experience a decline in total daily sodium intake (mg), decrease in systolic and diastolic BP (mmHg), and a reduction in the number of anti-hypertensive medications used. 


\section{METHODOLOGY}

\section{Design and Subjects}

This study served the purpose of documenting how low sodium focused, liberalized renal nutrition recommendations can affect dietary sodium intake, BP, anti-hypertensive medication, and prevalence of VO in U.S. HD patients. This is a retrospective analysis of a prospective, multi-center, intervention conducted in Central Illinois.

Purposive, convenience sampling was used to identify 32 HD patients from participating HD clinics in Peoria and Champaign, Illinois. Inclusion criteria was HD treatment for ESRD, and the exclusion criteria was decompensated heart failure as classified by a physician. Patients gave written consent for participation and signed health information release forms prior to starting, allowing for research staff to access medical information including height, weight, medications, HD and comorbidity history, and other laboratory reports. Following consent, all patients were evaluated by a registered dietitian and a nephrologist for safety clearance to participate. The University of Illinois and Illinois State University Institutional Review Boards approved this protocol. All procedures were in accordance of the Declaration of Helsinki.

\section{$\underline{\text { Data Collection Periods }}$}

The two data collection periods included the week prior to a patient starting the intervention, and the week following the conclusion of the six-month intervention treatment. During each week-long collection period, patients underwent a series of measures at their regular HD sessions including dietary intake, nutrition knowledge, BP, medication use, VO, and other related values. Collection was conducted by a trained member of the research staff or a HD technician or nurse. 


\section{$\underline{\text { General Measures }}$}

Data on demographic features were collected (age, gender, smoker), alongside clinical measures (height, post-dialysis weight, interdialytic weight gain (IDWG), presence of diabetes, HD vintage in years). Continuous variable measures were taken in triplicate and averaged where appropriate.

\section{Body Fluid and Volume Overload}

Evaluation of body fluid and fluid overload experienced by a patient was conducted by a trained researcher using a portable bioimpedance spectroscopy (BIS) machine (ImpediMed SFB7). Patients underwent this non-invasive procedure prior to and following HD treatment once during each collection period. For this measure, the BIS system utilized a single channel of tetra polar BIS to scan electrical frequencies for patient body composition estimation. Each patient was seated and had two electrode leads each placed on their non-access hand and foot on the same side of the body. The two hand leads were placed on the wrist next to the ulnar head, and on the dorsal surface of the hand. The foot leads were placed on the dorsal surface of the foot on the ankle at the level of medial and lateral malleoli, and on the dorsal surface of the foot. This low frequency BIS system assessed patient's extracellular fluid (ECF), intracellular fluid, and total body water (TBW). Based on these outputs, the patient's percent and liters of VO could be calculated. $^{22}$

\section{$\underline{\text { Blood Pressure and Medication Use }}$}

BP measures were taken by an HD technician or nurse during every HD session the patient attended. For this study, measures of pre-HD BP were recorded during each collection period, each on a separate HD treatment day. Prior to the start of HD treatment, patients had their BP measured through a sphygmomanometer attached to an HD machine. Patients were asked to 
sit resting for at least five minutes prior to testing BP on their non-dialysis port arm. Measures of systolic and diastolic BP were documented and the treatment commenced. Throughout the entirety of the study, patients had their systolic and diastolic pre-HD BP documented six times, three pre-intervention and three post- intervention measurements. Following both collection periods, systolic and diastolic BP were averaged to interpret the mean pre-intervention BP and mean post-intervention BP.

Patients self-reported how many anti-hypertensive medications they were currently prescribed from a physician and taking on a regular basis to help control elevated BP levels and HTN. To ensure accuracy, patients brought all current prescriptions to an HD session and research staff documented how many were for BP reduction. The number of anti-hypertensive medications a patient was prescribed was recorded once during each collection period. Nutrition Knowledge

Patients were evaluated on their knowledge of nutrition information about protein, sodium, and phosphorus prior to and following the intervention period. Questionnaires were based on label reading, nutrient content of common foods, and general knowledge related to nutrition. ${ }^{23,24,25}$ Each patient completed this questionnaire one time during each collection period Scores were calculated for percent correctness on each questionnaire.

\section{Dietary Intake}

Each patient underwent three 24-hour dietary recalls during each collection period for three separate days of food consumption. Recalls were conducted by a trained researcher based on the USDA 5-pass method with steps for documenting foods eaten, probing for forgotten foods or beverages, prompting for portion sizes, questioning for brand names, and final confirmation of the day's intake (Appendix A). This multi-pass approach collects a patient's single day dietary 
intake of food, beverages, vitamins, minerals, and supplements. The three recalls were evaluated on a dialysis weekday, non-dialysis weekday, and a non-dialysis weekend day. Each recall session lasted approximately 15 minutes. Dietary recalls were conducted verbally and recorded by hand then later transferred into the Nutrition Data System for Research (NDSR) software for analysis of micronutrient and macronutrient intake. Within the scope of this investigation, a total of six 24-hour dietary recalls were completed, three pre-intervention, and three post-intervention. After evaluating through NDSR, patient's dietary intake was consolidated to average preintervention intake and average post-intervention intake and used for analysis.

\section{Intervention}

\section{Liberalized Renal Diet Education}

Patients were counseled individually on the liberalized renal diet by a researcher twice per week for six months. Counseling sessions were held during a patient's regular HD sessions and each lasted approximately 15 minutes. Weekly counseling included individualized education, goal setting, and patient support focused on a liberalized renal diet and reducing patient sodium intake. Patients discussed their previous week's meals and adherence to the guidelines of a liberalized renal diet (Appendix B). Interviews served to continue discussion and education about the diet, and analyze the patient's progress towards achieving their personal goals. Dietary goal setting was conducted through a specific, measurable, attainable, relevant, and time-related (SMART) goal model, a validated and reliable method of developing clinically based goals $^{26}$ (Appendix C). 


\section{Volume Reduction Protocol}

In combination with reducing an individual's dietary sodium intake and BP medication use, the objective of the volume reduction protocol was to slowly decrease participants' postdialysis weight, thereby decreasing their degree of total VO and ultimately reducing their estimated dry weight (EDW). EDW was reduced until either the patient's BP normalized (below $140 / 90 \mathrm{mmHg}$ ) or the patient consistently experienced hypotensive events including cramping, headaches, or nausea during their HD treatments. All volume reduction efforts were conducted under the supervision of a nephrologist. Nurses and technicians were instructed to decrease the post-dialysis weight by 200-300 grams per dialysis session, until pre-dialysis BP measures normalized to $140 / 90 \mathrm{mmHg}$ or less in the absence of antihypertensive medications. HD staff were discouraged from administering saline to mitigate complications or discomfort, as were patients from requesting saline or a modification of their prescription.

\section{Statistical Analysis}

Statistical analyses were conducted using the Statistical Package for Social Sciences (SPSS) version 22 (Chicago, IL, USA). Continuous variables were reported as mean and standard deviation. Comparison of pre-and post-intervention measurements was conducted using the paired t-test. Categorical data was analyzed using the chi-squared test and are reported in frequencies and percentages. A p-value $<0.05$ was accepted as significant for all statistical analyses. 


\section{RESULTS}

\section{Participant Demographics}

Individuals with ESRD receiving maintenance HD at the Peoria, IL and Champaign, IL Fresenius dialysis clinics were invited to participate in the study. All 32 patients consented to participate, but nine individuals elected to discontinue the study protocol before completion, reducing the number of participants to 23 . Participants withdrew for various reasons including: relocation, transplant surgery, extensive health concerns, and imprisonment. Within the 23 participants, 12 were males and 11 were females between the ages of 22-81 years old (mean 55.7 \pm 13.3 years), of mixed ethnicity (Caucasian, African American, Hispanic) (Table 1).

\section{Body Fluid and Volume Overload}

Results from BIS allow for comparison of fluid volume and VO changes related to the intervention. Significant changes were seen for ECF decreasing from $21.7( \pm 5.8)$ to $20.5( \pm 4.5)$ $\mathrm{L}(\mathrm{p}=0.004)$ (Figure 1) and for VO values as well. VO in liters reduced from $3.6( \pm 3.9)$ to $2.5( \pm$ 3.5) L ( $\mathrm{p}=0.01)$ (Figure 1), a decrease from $15( \pm 15)$ to $11( \pm 17) \%(\mathrm{p}=0.04)$ (Figure 2$)$. TBW did not change with intervention $(45.8( \pm 10.8)$ to $44.3( \pm 8.8) \mathrm{L}(\mathrm{p}=0.10))($ Table 2$)$.

\section{Weight}

Weight measures for participants were compared following the intervention period, including average weight and average IDWG. A significant reduction was seen in patient weight on average from $93.6( \pm 24.4)$ to $91.8( \pm 22.7) \mathrm{kg},(\mathrm{p}=0.02)$, although the drop in average IDWG was negligible at $3.2( \pm 1.1)$ versus $3.0( \pm 1.0) \mathrm{kg},(\mathrm{p}=0.40)$ (Table 1). 


\section{Blood Pressure and Medication Use}

Mean pre-dialysis BP measures did not differ for both average systolic and diastolic values following the intervention. The non-significant alteration in systolic BP valued at $160( \pm$ $25)$ and $156( \pm 23) \mathrm{mmHg}(\mathrm{p}=0.56)$ for pre- and post- measurements, respectively, while diastolic BP was $81( \pm 20)$ and $79( \pm 15) \mathrm{mmHg}(\mathrm{p}=0.73)$. Although pre-dialysis BP measurements did not change significantly, anti-hypertensive medication decreased significantly after the 6-month intervention period. On average, anti-hypertensive prescriptions reduced significantly from $3( \pm 1)$ to $2( \pm 1)$ medications per participant $(\mathrm{p}=0.003)$ (Table 3$)$.

\section{Post Intervention Dietary Intake and Knowledge}

Mean dietary sodium intake, analyzed through 24-hour dietary recalls, decreased nonsignificantly following the intervention with measurements of 2,886 $( \pm 1,570)$ and 2,315 $( \pm$ $1,095) \mathrm{mg}$ at pre- and post-intervention, respectively $(\mathrm{p}=0.125)$. Additionally, protein consumption did not differ before and after the intervention period $(61 \pm 30$ before and $56 \pm 21 \mathrm{~g}$ protein after, $\mathrm{p}=0.60)$. No significant alterations were seen in energy intake per day before and after intervention $(1,588 \pm 1,061$ kilocalories (kcals) vs. 1,366 $\pm 455 \mathrm{kcals},(\mathrm{p}=0.38))($ Table 4).

Nutrition knowledge levels, measured through the percentage correct of questionnaire responses, reflect progression following the intervention. Significant improvement was seen for average phosphorus knowledge questionnaire scores from $66( \pm 15)$ to $74( \pm 14) \%,(p=0.04)$. Although non-significant, progression in sodium and protein knowledge was also seen. Average sodium scores increased from $68(+27)$ to $76(+30) \%(\mathrm{p}=0.06)$ following the intervention, and numerical changes in average protein knowledge scores ranged from $81( \pm 18)$ to $84( \pm 14) \%$, $(p=0.25) .($ Table 5). 


\section{DISCUSSION}

The main objectives of this study were to explore the impacts of a liberalized renal diet with volume reduction on HD patients. Areas of measurement included demographics, dietary intake, nutrition knowledge, BP, anti-hypertensive medication use, and body fluid levels. The primary new findings of this preliminary intervention study are that individuals receiving a liberalized renal diet education alongside volume reduction HD protocol had 1) reductions in VO; 2) decreases in average weight; 3) significant reduction in BP medication use with marginal differences in BP; 4) improvement in dietary phosphorus knowledge with slight increases in dietary sodium and protein comprehension; and 5) minimal variance in dietary sodium, protein, and calorie intake.

Body fluid levels deciphered through BIS were noteworthy on multiple fronts with decreases in total VO and ECF. Significant reductions in VO and body fluid retention can likely be attributed to the aggressive fluid removal that took place during volume reduction HD protocol. By continuously lowering a patient's target dry weight by 200-300 grams per session, their body fluid levels were predicted to gradually decrease. This decrease in body fluid may then lead to reduced CV workload, decreased BP, and less edema. ${ }^{21,27}$ Reduction in a patient's dry weight is beneficial as their dry-weight is often determined only by clinical judgement rather than an objective measure of actual patient weight without edema. ${ }^{9}$ A similar study described their fluid removal process as "intensified" and used maximal ultrafiltration (UF) on their patients until their BP was below $140 / 90 \mathrm{mmHg}$ without medication usage, ${ }^{21}$ with supplemental HD session as needed. Similar to our findings, the intensified UF resulted in decreased extracellular fluid and blood volume levels, likely benefiting $\mathrm{CV}$ health through reduced blood volume and stress on the CV system. Coinciding with the overall decreases in body fluid 
overload, patients also experienced a significant decrease in average weight following the intervention. As changes in weight for HD patients are often related to changes in body hydration status, this finding is likely correlated with the reduced VO status. ${ }^{9}$

A sodium restricted diet is an integral component to success with this modified UF protocol as it should decrease patient thirst and overall fluid intake. Ozkahya et al., ${ }^{21}$ also implemented a sodium restricted diet, and reported significant reductions in BP outcomes with little medication usage. Patients in the Ozkahya et al., ${ }^{21}$ study had high adherence rates to the low sodium diet protocol, which was contrary to the findings within our study. Indeed, other investigations reported reductions in sodium intake associated with decreased fluid retention, thirst, and BP. ${ }^{6,21,28}$

With non-significant reductions in sodium intake, contrary to the hypothesized outcome, the reported body fluid reductions are likely unrelated to decreases in sodium intake. Although the dietary protocol may not have greatly impacted body fluid levels, the overall decrease in fluid overload may in turn reduce patient mortality rates $^{22}$ and could improve the ability to decipher a patient's actual EDW. ${ }^{29}$

As decreases in body fluid overload are related to BP reduction within HD patients ${ }^{9,28}$, we anticipated a resulting decrease in BP for our patients alongside their reduced VO. Contrary to expectation, our participants did not have a significant reduction in systolic nor diastolic BP. The negligible change seen in BP levels may have been due to poor adherence to the low-sodium dietary protocol, as other studies with persistent UF and low sodium diets found success gaining control of BP levels following similar interventions. ${ }^{16,21}$ Due to the minimal sodium restriction seen, we hypothesized that our participants did not realize the full extent of possible HTN attenuation. With substantial reductions in sodium, our participants could have experienced 
better VO control, favorable improvement in BP control, and greater preservation of their systolic and diastolic functions with little to no reliance on BP medications. ${ }^{7}$

Although BP adaptations were not prominent, reduction in overall VO did seem to have a positive effect on anti-hypertensive medication uses. On average, our participants significantly decreased their medication usage as hypothesized without seeing a consequent rise in BP, indicating some improvement in BP control. Though BP levels were stagnant, diminished patient dependency on medications may help reduce other concerns such as cardiac stress and mortality rates, as well as producing fewer cases of intradialytic hypotension, with decreased severity of left ventricular dysfunction. ${ }^{15,16}$ Our patient's decrease in body fluid overload likely lowered their BP medication needs, while avoiding a subsequent spike in BP. Although there was no significant change in BP, it can be hypothesized that with greater low sodium diet adherence, patients on a similar UF protocol would experience a decrease in both anti-hypertensive medication usage and BP.

Renal dietitians are the primary providers of nutrition education for HD patients. ${ }^{14}$ In previous interventions, patients received dietary education materials to foster adherence to a therapeutic diet. ${ }^{30}$ In our intervention, we attempted to give customized nutrition education to patients through individual counseling sessions. With additional knowledge of nutrition concepts, we predicted that individuals in this intervention would adapt their dietary intake based on guidelines provided through education modules. Post-intervention, participants displayed significantly higher phosphorus knowledge scores, with some improvements in sodium and protein knowledge.

With the nutrition knowledge progress, the outcomes for dietary sodium, protein, and calorie intake did not correlate with our prediction. Individuals experienced non-significant 
decreases in general across these dietary intake categories, yet only sodium reduction was specifically targeted in participant counseling sessions. As sodium reduction was a primary marker of patient dietary adherence, the non-significant reduction highlights a lack of compliance from participants that may have been instigated by barriers for dietary change. Difficulty adhering to a reduced sodium diet is not unheard of, as previous investigations have indicated multiple barriers HD patients face when limiting sodium intake. Challenges for reducing sodium intake include limited availability of low sodium foods, poor intrinsic motivation for dietary change, and limited understanding of dietary change and associated health outcomes. $^{13,33}$

One prominent factor making sodium reduction so difficult is the lack of social support patients get from their family and friends. ${ }^{13,31}$ Patients often feel uncomfortable discussing their special dietary needs with their loved ones, and their family is not often involved with education sessions, limiting the family's ability to assist or participate in the dietary changes. Consideration for the barriers to understanding and achieving a low sodium diet may be necessary to clarify the importance of the diet and encourage inclusion of loved ones in this lifestyle change.

Additionally, sodium is a component of many food items sold commercially and avoiding high salt foods can be almost impossible. Both patients and professionals agreed that avoiding sodium in pre-made foods is hard to do and time consuming. ${ }^{13,32}$ Furthermore, with the increasing use of sodium in foods for flavor and preservation ${ }^{32}$, many individuals have strong taste preferences for high sodium foods and they often do not find low sodium foods palatable. Although increases in knowledge may be associated with improved therapeutic diet adherence ${ }^{34}$, this may be a weak relationship compared to other contributing factors of dietary compliance. ${ }^{35}$ 
Neither protein nor kilocalories were recommended for reduction within the liberalized renal diet, thus patient's average numerical decrease in energy and protein intake was undesirable. Anorexia is a common barrier seen within the HD population and may have prompted the slight decrease in patient consumption. ${ }^{36}$ The minimal decreases may have also been related to the sodium restricted diet approach, as previous research indicates patients may decrease total energy intake to minimize sodium intake. ${ }^{11}$

Intake documentation utilized 24-hour dietary recalls that require an administrator to collect patient self- reported data, which allows low literacy patients to still participate. Dietary recall periods following closely after meals also reduces the risk for inaccuracies. ${ }^{37}$ Nevertheless, patient's self-report of intake presents opportunity for inaccuracy due to over reporting of healthy behaviors, under reporting of unhealthy behaviors, changes in behaviors due to the research project, inaccuracies based on a participant's ability to report correct information, or other unpredictable errors. ${ }^{38}$ Alternative methods for monitoring intake exist, but also have weaknesses such as generalizing patient food intake and lack of food choices presented in the recall ${ }^{37}$ Although the 24-hour dietary recall was the best choice for our intervention, the unpredictable level of error from patient self-reporting may have skewed the dietary intake results. Finally, our participants did spend time with our nutrition research staff during their HD treatments, but they did not spend additional time with their clinic's renal registered dietitian (RD). Increasing time spent with their regular renal RD may have produced different dietary outcomes.

Although participants did not achieve all the predicted outcomes, this study design did result in various improvements such as a decrease in anti-hypertensive medication use and significant reductions in VO. Some of the study's strengths included a dietary education 
approach based on individual goals meant to improve specific patient outcomes, and the gradual adaptation of an individual's estimated post dialysis weight through volume reducing UF. Our volume reduction process allowed for continuous decreases in each patient's post dialysis weight and re-evaluation of a patient's EDW, which likely impacted the positive decline in VO.

Alongside those strengths, areas for improvement and future research opportunities were also highlighted including: identifying and targeting personal barriers for dietary success, pursuing sustainable and self-motivated dietary goals, seeking to build an individual's support systems for greater dietary adherence, and eliciting greater support for this intervention from HD clinic staff.

The most prominent limitation of this study included a lack of staff participation and involvement within the selected HD clinics. Despite attempts to provide information to staff regarding the study, the intervention remained an effort conducted by outside research personnel. The regular dialysis staff lacked incentive for participation and were already busy. In order for this intervention to be truly effective, all HD care providers must be motivated to continually display a united message or risk blunted study outcomes. In future interventions, adequate training programs and communication techniques should be implemented. Attention to HD staff training may improve support and decrease confusion for all dietary and UF protocols and encourage all staff to be involved and accountable for patient care.

Although this study was a positive addition to the body of work surrounding a liberalized renal diet and volume reduction protocol, further research is needed in this field to ensure HD patients the highest care and quality of life. As many patients experience obstacles keeping them from making lifestyle changes, finding greater understanding of each individual's barriers to change may be beneficial for future studies. By identifying and catering a patient's treatments to 
their needs, a patient may see increased dietary adherence and improved health outcomes. Future interventions may also consider methods of nutrition education outside of the dialysis clinic. Presuming internet accessibility, exploring online instruction or video chats may allow for inclusion of family and friends in education sessions as well as allowing for alternate locations and learning resources. Removing lessons from the dialysis center may encourage greater patient comprehension and adherence, while offering the chance to build a patient's support system, a common concern for HD patients. ${ }^{14,33}$ Further studies should also consider additional lesson planning or training modules to educate all dialysis center and research staff on communication and appropriate research protocols. By creating open dialogue between the research and dialysis center staff, the ultimate goal of providing excellent patient care can better be achieved in addition to providing adequate research outcomes.

\section{CONCLUSION}

Patients experiencing liberalized renal diet education as well as volume reduction HD protocol decreased their average anti-hypertensive medication usage, and significantly reduced their VO. Although systolic and diastolic BP were unaltered contrary to the hypothesis, the stability of BP with a coinciding decrease in anti-hypertensive medication usage supports the consideration of lifestyle and UF changes as opposed to pharmaceutical approaches. This study provides preliminary evidence for future investigations aimed at lowering HD patient BP through sodium reduction and UF changes rather than prescription medication use. 


\section{CHAPTER II: LITERATURE REVIEW}

\section{INTRODUCTION}

End Stage Renal Disease (ESRD) has become an increasing public health concern, with over 110,000 new cases diagnosed in the United States (US) each year. ${ }^{1}$ ESRD refers to stage V chronic kidney disease (CKD), a disease state in which a patient experiences little to no kidney function. One role of healthy kidneys is to filter blood and remove excess fluid and metabolic waste from the body, and with inadequate kidney function, that ability is impaired. ${ }^{39}$ Without working kidneys, excess fluid, waste, minerals, and metabolites are poorly excreted through urine, influencing accumulation in the body. ${ }^{39}$

Metabolite and fluid accumulation leads patients with ESRD to require intensive medical care, including dialysis treatments to filter the patient's blood in absence of adequate kidney function. ${ }^{1}$ Two primary variations of dialysis treatment include peritoneal dialysis, and hemodialysis (HD), with HD being the most frequently selected treatment. ${ }^{39,40}$ In the US, HD is typically a four-hour treatment that patients receive in a specialized facility, three times weekly. ${ }^{39}$ HD serves to remove the excess substances built up in the patient's body that would have otherwise been excreted as urine under normal circumstances.$^{39}$ During the time between dialysis sessions, also known as the interdialytic period, excess minerals and organic metabolites accumulate in the body, which can cause disturbances in the body's fluid status. Within HD patients, expansion of blood volume, and large interdialytic fluctuations of total fluid volume between HD treatments can increase or exacerbate health risks including cardiovascular diseases (CVD) such as hypertension (HTN). ${ }^{7}$ With upwards of $80-90 \%$ of HD patients experiencing $\mathrm{HTN}^{8}$, and CVD being the main cause of death among ESRD patients encompassing $41 \%$ of patient deaths ${ }^{10}$, prevention and treatment strategies are needed to overcome the HTN and CVD 
risks HD patients face. Common approaches to alleviate the negative health impacts of HTN in the HD population include various lifestyle changes and pharmaceutical approaches.

\section{LITERATURE REVIEW}

\section{The Traditional Renal Diet}

High mortality rates secondary to CVD within the HD population are concerning and dietary approaches are often recommended for HTN and CVD risk control. Interdialytic blood metabolite accumulation is a hallmark of the disease, upsetting fluid balance in the body. Traditionally, renal diet recommendations promote dietary mineral restrictions in hopes of preventing irregular blood mineral concentrations and fluid shifts. These traditional renal diet guidelines are quite restrictive, with limitations focused on sodium, potassium, and phosphorus intake for HD patients. $^{3}$

\section{Dietary Sodium}

In terms of HTN and CVD, a primary nutrient of concern is sodium. Sodium is a mineral and electrolyte important for fluid regulation and blood pressure (BP). Sodium is the principle extracellular cation, and is a strong moderator of water absorption and retention, with high sodium levels correlated to water retention. ${ }^{41}$ For HD patients unable to excrete excess fluid waste appropriately, any consumed sodium and in turn retained water will increase blood volume. ${ }^{41}$ This increase in blood volume then elevates BP, leading to development or exacerbation of HTN. In addition to sodium's strong ability to retain water, it also plays a role in thirst. Thirst for fluids is a physiological stimulus that can be increased if the body contains excess sodium levels. ${ }^{6}$ This stimulus is so strong, asking an individual to limit their fluid intake without instructing them on sodium limitations has even been considered inhumane. ${ }^{6}$ Because HD patients are limited in their fluid waste excretion, any additional fluid intake will impact their 
blood volume, and can lead to cardiovascular (CV) concerns including HTN and aortic stiffness ${ }^{7}$, supporting the need to restrain thirst. Because sodium causes the body to retain water, and increases an individual's thirst and drive to consume liquids, high sodium intake can drastically alter blood volume and pressure. ${ }^{41}$ In an investigation with greater than 30,000 individual HD patients, large gains of body fluid between dialysis sessions, more than $4.0 \mathrm{~kg}$, were correlated with higher risk of death over the two years following ${ }^{9}$, indicating the need for interventions. HTN rates are so elevated, effecting an estimated $80-90 \%$ of $\mathrm{HD}$ patients ${ }^{8}$, and CVD risk is rampant for HD patients. As blood volume overload (VO) is predicted as one of the most frequent components of HTN progression ${ }^{27}$, there is a strong argument for sodium restriction within this population.

Based on this concept, adjusting sodium intake has been investigated as a treatment for fluid retention and HTN within the HD population. A study by Maduell and Navarro ${ }^{17}$, attempted to reduce dietary sodium intake in HD patients. Decreasing sodium intake led to significant reductions in patient's systolic and diastolic BP. From this 15 -subject study, researchers found sodium limitations to be an important controller of HTN in HD patients, and found sodium restrictions to be more effective than antihypertensive medications for lowering $\mathrm{BP} .{ }^{17}$

Although sodium restrictions could be a strong determinant of fluid balance and HTN in HD patients, there are challenges to decreasing sodium intake. Sodium is used as a preservative for many processed food products and is highly abundant in restaurant foods as well. ${ }^{32} \mathrm{As}$ most foods are naturally low in sodium, foods that have been modified or processed prior to consumption are the most common source of sodium in the American diet. With an abundance of sodium available for US consumers, most Americans meet and exceed daily recommended sodium intake for adults. ${ }^{32}$ Increased access and intake of sodium rich foods creates concern in 
regards to elevated body fluid levels, and CVD risk for HD patients is greatly increased with excess sodium intake. Elevated body fluid poses a serious CV threat to HD patients, and due to sodium's strong correlation with overall fluid retention, considerations should be make regarding sodium intake for HD patients.

\section{Dietary Potassium}

Another nutrient of concern that can accumulate in the blood between HD sessions is potassium. Traditional renal diet recommendations include guidelines for potassium intake to discourage accretion of potassium in the blood.$^{39}$ Hyperkalemia, excess blood potassium, is the most common metabolite abnormality for HD patients. ${ }^{42}$ Hyperkalemia is associated with higher risk of $\mathrm{CV}$ mortality for $\mathrm{HD}$ patients ${ }^{43}$, making alterations in dietary potassium and other medical treatments a focus for HD patients to achieve optimal blood potassium levels.

Dietary potassium limitations are traditionally recommended for HD patients to support potassium balance and decrease hyperkalemia risk. However, strict potassium limits can pose concerns for various food groups due to the high potassium concentration in many nutrient rich foods. Some foods that are naturally high in potassium include fruits, vegetables, nuts, and legumes, foods that are also notably high in fiber and beneficial nutrients. ${ }^{44}$ Potassium is also used as an additive to many products including soft drinks and bread, illustrating how difficult avoiding dietary potassium can be. ${ }^{45}$ Decreasing hyperkalemia risk is still essential to reducing CVD risk, but restriction of all potassium foods may not be necessary. Data linking naturally potassium rich foods to adverse health outcomes is limited. ${ }^{43}$ Furthermore, foods with potassium added for preservative or flavor purposes often have low nutrient density and are not typically favorable selections. ${ }^{20}$ With limited research discussing the benefits and downsides of natural potassium in nutrient rich foods, these recommended limits may be unnecessarily strict. 
Additionally, as foods with added potassium are often nutrient poor, these foods should likely be the initial target when removing potassium containing foods from the diet. Continuing research is pursuing investigation of what quantity of natural and added potassium is safe for HD patients. ${ }^{20}$ Although a patient may require potassium intake limitations, restricted consumption of foods naturally containing potassium may be less necessary than previously indicated.$^{20}$ With limited data supporting dietary potassium restrictions, fresh produce and other natural potassium containing foods may still be safe for HD patients. Further evidence is needed to determine appropriate potassium recommendations for HD patients.

\section{Dietary Phosphorus}

Phosphorus, similar to potassium, is another mineral causing concern for HD patients with the potential of hyperphosphatemia, or high blood phosphorus ${ }^{46}$, due to patient's limited excretion of phosphorus through urine. Limitations on all dietary phosphorus are traditionally noted as a preventative method for HD patients to avoid hyperphosphatemia, but recently, this ideology is being challenged. ${ }^{47}$

Foods that are naturally high in phosphorus include milk, cheese, beans, and meat ${ }^{42}$, with other foods such as carbonated sodas and processed foods being high in phosphorus from additives and preservatives. ${ }^{32}$ Research indicates that inorganic phosphorus added to foods during processing has up to $100 \%$ absorption rates within the gut, in comparison to the $40-60 \%$ of natural phosphorus that is absorbed from unprocessed foods. ${ }^{47}$ Although absorption rates differ, dietary recommendations do not decipher between naturally phosphorus rich foods and those with added phosphorus from processing. ${ }^{47}$ Furthermore, natural phosphorus is often present in foods with higher levels of protein and/or fiber, indicating extensive dietary phosphorus 
limitations may be harmful to an individual's ability to consume adequate protein ${ }^{47}$, a consistent concern for this population. ${ }^{36}$

In efforts to avoid hyperphosphatemia, while maintaining adequate protein and energy intake, avoidance of phosphorus additives and processed foods is indicated, without limitation on natural phosphorus and protein rich foods. ${ }^{47}$ This method will allow patients greater food variety and could improve patient protein intake, common HD patient concerns. ${ }^{14}$ Additionally, patients may have improved health outcomes as more restrictive phosphorus recommendations have been associated with higher mortality rates among HD patients. ${ }^{46} \mathrm{With}$ research indicating differences between added and naturally occurring phosphorus, patients may be able to avoid hyperphosphatemia by eating foods such as meats, beans, and dairy, while actively avoiding added phosphorus within processed foods.

\section{Traditional Renal Diet Challenges}

The traditional renal diet has been described as one of the most challenging diets a dietitian can prescribe to patients ${ }^{11}$, including limitations on sodium, potassium, and phosphorus. Unfortunately, this restrictive diet produces limited health outcomes in terms of HTN and CVD. Previous studies have investigated guidelines for limiting mineral consumption for HD patients, often noting foods such as beans, root vegetables, and fruits as high in unhealthy minerals. ${ }^{44}$ In addition to the general challenge of attempting to understand and achieve these strict dietary goals, further barriers are seen in terms of processed foods. Many patients are not educated on levels of minerals present in highly processed food items, with process foods being defined as those items that have been deliberately changed in some way from the time of origin to the time of consumption. ${ }^{48}$ Processed foods may not be on the exclusion list traditionally given to HD patients, but these foods likely contain unhealthy amounts of minerals. ${ }^{32}$ Additionally, processed 
foods are more often insufficient in fiber and essential nutrients, components HD patients traditionally lack within their diet, while still containing astonishingly high amounts of sodium. ${ }^{12}$ Traditional renal diet guidelines are currently limiting patient food options, while being unclear about the use of processed foods and the sodium content that may be hiding within these products. ${ }^{32,49}$ With the expansive presence of processed foods and mineral additives used for flavor and preservation, identifying and avoiding foods high in sodium, potassium, and phosphorus can be difficult..$^{32,44}$

With traditional renal dietary limitations in place, HD patients are also particularly prone to suffering from protein-energy wasting ${ }^{11}$, including multiple nutritional and catabolic alterations that occur alongside kidney disease and failure. ${ }^{36}$ By reevaluating these past guidelines, a patient may be able to improve their nutrition status with liberation of phosphorus intake and inclusion of the previously limited dairy and meat food groups. ${ }^{47}$ Moreover, guideline reconsideration may improve the vitamin and mineral deficiencies HD patients typically display $^{11,50}$ with more freedom regarding fruit and vegetable intake, previously limited due to high potassium concentration. ${ }^{14,44}$ With indication that naturally-occurring potassium and phosphorus rich foods may be safe for patients to consume, some guidelines of the traditional renal diet may be less necessary than previously thought.

Additionally, the complex guidelines of the traditional renal diet may detract attention from the sodium limitations patients likely need to focus on to improve their HTN and CV risk. Extensive dietary guidelines can incur poor dietary adherence based on a patient's ability and desire to pursue behavior changes. This includes patient differentiation in terms of cognitive abilities, cultural norms, and perception of potential health outcomes. ${ }^{51}$ When delving further into this issue, difficulties with social support, inadequate guidance offered to the patient by a health 
care provider, and limited patient understanding of disease state increasingly limits dietary adherence. ${ }^{13,14}$ As these guidelines are extensively complicated, some patients are unable to comprehend the information necessary for success. Also, patients who are unable or unwilling to adhere to dietary recommendations will not achieve highly on this restricted diet. ${ }^{13,14}$ HTN and excessive fluid retention are discussed in many research articles and the use of dietary sodium restriction is often noted..$^{5,15,17}$ These articles strongly advocate for limitations in dietary sodium, but provide little information as to how the process of moving towards a low sodium diet may work. Additionally, they lack documentation as to the potential change in BP and antihypertensive medications that may ensue should an individual limit their sodium intake. Though potentially applicable to American HD patients, most related research is conducted outside of the US. There is a possibility for the positive sodium restricted diet outcomes seen in Italy, Turkey, and France ${ }^{7,15,16}$ to also take place within the US, but further research is needed to confirm. Research is also needed to investigate the process of limiting sodium and the related outcomes, which will be relevant in future research for patients and practitioners to understand sodium restriction benefits for HD patients.

The challenges identified with the traditional renal diet make it difficult to pursue, and the identified weakness of its recommendations truly contest the necessity of this diet. In efforts to create a diet that patients can understand and achieve when they are ready for behavioral modifications, further investigation is warranted to determine meaningful and attainable dietary recommendations for HD patients. 


\section{Anti-Hypertensive Medications}

As discussed previously, BP control is crucial for HD patients to maintain their CV health due to their limited liquid waste excretion and blood metabolite build up. In addition to diet or lifestyle changes, pharmaceutical treatment approaches are an option to achieve recommended BP levels.

Anti-hypertensive medications are identified as the most common method of treatment for $\mathrm{HD}$ patients with $\mathrm{HTN}^{5}$, with multiple types of anti-hypertensive medications being prescribed individually or in tandem. ${ }^{27}$ With many classes of medications available, patients can receive multiple prescriptions with varying outcomes depending on their $\mathrm{CV}$ health, comorbidities, and physician. In general, the US is one of the highest users of anti-hypertensive agents.$^{52}$ Poly-pharmaceutical therapies are so common, that amongst a group of 205 American HD patients taking antihypertensive medications, $58 \%$ of those individuals were taking two or more medications daily. ${ }^{18}$

With a poly-pharmaceutical approach in place, improved BP outcomes would be expected, but medication based therapies often have limited effects on BP. In a study of 2535 HD patients prescribed one or more anti-hypertensive medications, 55-75\% of patients remained hypertensive above $140 \mathrm{mmHg}$ for their systolic $\mathrm{BP} .{ }^{27}$ Limited outcomes for BP management may be related to the varying etiologies of HTN, as patients with HTN unrelated to blood VO seem to fair better to the treatment, as opposed to those individuals who have excessive blood volume impacting their $\mathrm{BP} .{ }^{27}$ As many HD patients have VO contributing to their HTN, the likelihood of success with anti-hypertensive medications is limited. It is likely that a treatment reducing total blood VO will have greater impact on HD patient BP than altering other targets. ${ }^{27}$ In addition to inadequate BP reduction outcomes, pharmaceutical approaches have challenges 
including cost, side effects, and initiation and maintenance of medication routines. For patients, unfamiliar or unable to access affordable medication options, adherence and maintenance to the medication regimen may be difficult. Furthermore, some patients challenged with handling their own care including continuous medication usage can increase concern for misuse or poor medication adherence due to limited ability to care for themselves and maintain medications. ${ }^{5,15,18}$ Although medications are a commonly prescribed approach for HTN reduction, the outcomes are limited and use is challenging for numerous HD patients, indicating need for further HTN treatment consideration.

\section{Ultrafiltration Techniques}

The process of HD is an ultrafiltration (UF) of a patient's blood to remove excess waste and fluids retained between dialysis sessions. Traditional US HD processes are successful in removing some excess fluid and metabolites from the blood based on a patient's estimated dry weight (EDW). The EDW is predicted at each dialysis session by a nephrologist, and is based on the practitioner's best approximation of a patient's weight if they did not have any excess fluid retention. ${ }^{9,21}$ Although these estimations can be close, the ability for the practitioner to accurately predict every patient's EDW, every session, is unlikely. Even with substantial levels of human error, limited options for predictive methods have led to EDW being the standard approach for nephrologists writing UF prescriptions. ${ }^{9}$ Although traditional HD approaches for American patients use EDW quite consistently ${ }^{39}$, some researchers have discussed altering UF approaches to improve BP and VO outcomes. Many of the most successful approaches to decreasing HTN in HD patients includes using extended or more frequent HD sessions, allowing for greater fluid removal and blood volume management. ${ }^{27}$ Another investigation looked at providing four-hour HD sessions removing as much fluid as the patient could safely tolerate, with additional HD 
sessions as indicated for elevated blood volume and $\mathrm{BP} .{ }^{21}$ This approach did not utilize EDW to determine the UF prescription, but instead used UF to consistently decrease BP without causing severe instances of hypotension or other contraindications to fluid removal. ${ }^{21}$ Through this technique more fluid was removed allowing patients to come closer to a status of little to no fluid retention. With this personalized approach to the UF protocol, patients in this study saw decreases in cardiac volume, and BP control was more easily achieved, leading researchers to expect improved CV outcomes. ${ }^{21}$ Further reinforcement for individualized approaches to HD treatments are recommended due to the fine precision available through HD technology currently used at HD clinics. With the ability to create unique HD prescriptions based on a patient's individual needs at the time of their session, opportunities for improving BP and fluid balance are available ${ }^{53}$ Unfortunately, many HD clinics note difficulties regarding cost and staff training levels as reasons to not pursue unique and adaptive HD prescriptions ${ }^{53}$ With variations of UF treatments indicating potential improvements for BP and CVD risk, HD patients with HTN may be good candidates for alterations in the UF prescription as achievable to provide improved health outcomes.

\section{Comparing Hypertension Management Techniques}

Due to the high prevalence of HTN and CVD in HD patients, determining appropriate treatment approaches is vital to patient health outcomes. With distinct differences between dietary changes, pharmaceutical approaches, and UF techniques, pinpointing the best treatment plan can be puzzling. Some HD clinics are making transitions in their daily patient care in efforts to reduce $\mathrm{BP}$.

Conversion from a medication based therapy for HTN to sodium restriction and UF therapy has taken place overseas in countries such as France. In a comparison of two respected 
European HD centers, a French clinic had distinguished differences in survival rates and BP. ${ }^{15}$ Investigating the mortality among patients at these clinics, the survival rate is markedly higher at the French HD center, hypothesized to be related to the low prevalence of HTN among the patients. The French clinic functions on a reduced sodium diet approach for BP regulation accompanied by extended eight hour HD sessions to decrease blood VO. The English clinic recommends a medication based approach for HTN with traditional four hour HD treatments, similar to that of the US. With superior life expectancy outcomes for the French patients provided low sodium diet recommendations and altered HD treatments, this research may call for reevaluation of a medication based approach. In this case, sodium restriction and UF changes in the French clinic seemed to decrease HTN as well as mortality rate, whereas anti-hypertensive medications seemed to make less impactful differences, if any at all. ${ }^{15}$ For comparison to the US, total sodium intake for the patients at the French clinic was about 1700 milligrams per day ${ }^{15}$, with American patients consuming an average of 4200 milligrams of sodium daily ${ }^{54}$, indicating opportunity for sodium intake improvements. This study's findings are not alone, as similar outcomes are seen in Turkish HD centers.

In a comparison between Turkish and American dialysis patients, individuals were evaluated for HTN as well as mortality rates. For hypertensive US HD patients, medications are often the primary treatment, alongside recommendations for the traditional renal diet. However, Turkish patients follow approaches to reduce over hydration with more aggressive fluid removal during HD, as well as a strict dietary sodium restriction without the potassium and phosphorus limitations of the traditional renal diet. The combination of aggressive fluid removal during dialysis and focusing dietary recommendations solely on sodium intake appears to improve BP in most Turkish patients. With a 50\% lower mortality rate among Turkish patients, it is likely 
that Turkish approaches to blood VO and sodium limitations are more effective than those used in the US. ${ }^{55}$

One of the few comparison studies, Kayikciogly et al. ${ }^{16}$, tracked hypertensive HD patients at two separate dialysis clinics treated with either a low sodium diet or anti-hypertensive medications and compared BP outcomes. The 423 HD patients involved in this Turkish study all received the same HD treatment approach. Individuals at Center A were actively recommended a 2000 milligram sodium diet daily for HTN, rather than being passively recommended a reduced sodium diet and relying on anti-hypertensive medications provided at Center B. At Center A, dietary sodium restriction indicated less strain on patient's left heart ventricle indicating better function, lower risk of hypotension following HD treatments, and improved systolic and diastolic function. Additionally, patients adhering to a sodium restricted diet experienced lower interdialytic weight gain (IDWG) between dialysis sessions than those only taking antihypertensive medication $^{16}$, and notably lower IDWG is associated with greater survival rates. ${ }^{9}$ Following the conclusion of this trial, the positive CV outcomes indicate the therapy from Center A, using sodium restriction, was more beneficial. ${ }^{9}$

Further investigations to decrease total blood volume and fluid retention with sodium limitations had similar findings. Within a study of 15 HD patients, a dietary sodium limitation was an important controller of HTN. These individuals experienced decreases in both systolic and diastolic BP through the dietary sodium restriction, and the dietary lifestyle approach was considered more effective than medication use, though no medications were compared within this study. ${ }^{17}$ An additional case discussing sodium restriction and BP regulation for HD patients, used an intervention approach to decrease dietary sodium intake from an average of 4.1 grams of sodium per day to 2.8 grams of sodium per day. This intervention aided in significantly reducing 
patient systolic $\mathrm{BP}^{54}$, further supporting this platform. This strong viewpoint contradicts the current popularity of anti-hypertensive drugs for HD patients. ${ }^{5}$ But although the transition from medication based treatments to dietary approaches may seem beneficial, barriers to transitioning from a pharmaceutical approach to a low sodium diet are still impacting patient success.

\section{Dietary Sodium Reduction Challenges}

Regardless of the strong research supporting use of a low sodium diet for BP control in HD patients, there are continued personal and cultural barriers to this lifestyle. Studies often note the challenges of following a sodium restricted diet including; inadequate access to low sodium foods, lack of self-motivation or family support, varying dietary beliefs, personal attitudes, and poor taste acceptance. ${ }^{13,31}$ With patients experiencing universal barriers to lifestyle changes as well as those unique to them as an individual, finding approaches to overcoming these obstacles is vital to patient success. Recent literature has considered ways to improve patient adherence, with a large focus on increasing accessibility to lower sodium foods for patients following this diet.

Sodium is often used in food processing as a preservative or to improve food taste. ${ }^{32}$ Foods naturally contain relatively low amounts of sodium, but approximately $75 \%$ of American sodium intake comes from sodium added during processing..$^{32}$ In efforts to control sodium additions in processed foods, one study experimented with minimizing the sodium content of common foods. ${ }^{56}$ Researchers decreased sodium additions by an estimated $50 \%$ in almost all food categories and indicated that this would result in a $38 \%$ decrease of daily dietary sodium intake for most individuals. ${ }^{56}$ Similarly, guidelines for maximum values of sodium additions are being considered in efforts to decrease sodium consumption in America. ${ }^{49}$ These guidelines would limit processing companies' ability to exceed the maximum sodium amount designated, 
and may decrease individual's daily sodium intake..$^{49}$ It appears there is a great possibility to change within the food industry that may benefit the sodium restrictions needed by HD patients.

Some concerns that may arise from the low sodium diet approach have been noted by researchers, including risk of undernutrition. Some studies indicate that individuals may not receive adequate amounts of micro and macro nutrients while on a limited sodium diet. ${ }^{11}$

Although it can be challenging to find some low sodium food alternatives, it appears that patients can adequately achieve energy and protein intake with appropriate alternative food selections. ${ }^{11,57}$ Additionally, considering the other option of the restrictive traditional renal diet, the low sodium diet approach will likely provide more nutrients than the alternative. Further success on the low sodium diet can be found with maintenance of protein intake, as that indicates increased survival rates. ${ }^{11}$ With consideration for nutrients at risk while on a sodium restricted diet, patients can improve their HTN by consuming an adequate diet, specifically rich in protein and fulfilling energy needs.

By using low sodium and minimally processed products, an individual can successfully lower their sodium intake, which can decrease BP, while still maintaining health. This sodium limitation approach is not always easy to follow, but may be a more successful alternative therapy to anti-hypertensive medications.

\section{Reconsidering HTN Treatment in the US}

Research regarding sodium restriction for decreasing HTN among HD patients is not unheard of, but it appears the US is struggling to include that concept into practice. Medications are the most common treatment for HTN within the US. Unfortunately, with medication usage, the effects on BP within this population seem to be limited, with less than half of patients able to regaining control of their $\mathrm{BP} .{ }^{58}$ Not only is this approach minimally effective for patients, but it 
may dissuade the introduction of a dietary approach that could have more effective outcomes. If US HD clinics start to approach HTN treatments with a dietary sodium limitation, patients may be able to experience outcomes similar to those in France and Turkey, with lower BP as well as decreased left ventricular stress and reduced patient mortality rates. ${ }^{15,16,54,55}$ Additional opportunity for improvements may be seeded within the UF protocol and prescriptions provided to HD patients, allowing for greater individualization opportunities..$^{9,21,58}$

Recent research developments challenge the details of the traditional renal diet, as well as support dietary sodium changes versus medication based HTN management. Based on current research, reconsideration of these guidelines should focus on diet achievability and patient needs, while being conscious of minerals and providing adequate nutrients. ${ }^{11,20}$ These proposed dietary recommendations would include limiting foods high in sodium additives such as processed or restaurant foods. Additionally, this diet would allow more freedom for foods that are naturally rich in potassium and phosphorus. By focusing a patient's energy on reducing sodium instead of the traditional renal diet, a patient will have greater food selections and may increase their ability to find success achieving dietary changes. With the reconsideration of the traditional renal diet and stronger focus on dietary sodium reduction, patients may experience positive CV health outcomes, and decreases in systolic and diastolic BP, potentially even enough to warrant reduced anti-hypertensive medication use. Utilization of this low sodium focus may be further improved through individualized UF protocol, focused on improving a patient's VO and BP status, rather than on EDW. As investigations are limited regarding this low sodium diet focus and unique UF prescriptions within the US, further research should be considered to allow HD patients opportunities to achieve the beneficial health outcomes seen through low sodium diet and individual UF approaches in other countries. 


\section{REFERENCES}

1. Incidence, Prevalence, Patient Characteristics, and Treatment Modalities. https://www.usrds.org/2015/view/v2_01.aspx. Published 2016. Accessed October 15, 2016.

2. Stage 5 Kidney Disease - End-Stage Renal Failure I National Kidney Center. http://www.nationalkidneycenter.org/chronic-kidney-disease/stages/stage-5/. Accessed July 28, 2017.

3. Dietary Guidelines for Adults Starting on Hemodialysis. The National Kidney Foundation. https://www.kidney.org/atoz/content/dietary_hemodialysis. Published December 24, 2015. Accessed October 26, 2016.

4. Renal Support Network: Understanding Blood Pressure \& Dialysis. http://www.rsnhope.org/health-library/article-index/understanding-blood-pressuredialysis/. Accessed July 28, 2017.

5. Gull Z, Anwar Z, Sherazi BA. Management of Hypertension and Anti-Hypertensive Drug Profile in End-Stage-Renal-Disease Patients Undergoing Maintenance Hemodialysis. Medical Channel. 2016;22(3):41-46.

6. Tomson CRV. Advising dialysis patients to restrict fluid intake without restricting sodium intake is not based on evidence and is a waste of time. Nephrol Dial Transplant. 2001;16(8):1538-1542. doi:10.1093/ndt/16.8.1538.

7. Cupisti A, Aparicio M, Barsotti G. Potential Benefits of Renal Diets on Cardiovascular Risk Factors in Chronic Kidney Disease Patients. Renal Failure. 2007;29(5):529-534. doi:10.1080/08860220701391670. 
8. Iseki K. Control of hypertension and survival in haemodialysis patients. Nephrology. 2015;20(2):49-54. doi:10.1111/nep.12358.

9. Kalantar-Zadeh K, Regidor DL, Kovesdy CP, et al. Fluid Retention Is Associated With Cardiovascular Mortality in Patients Undergoing Long-Term Hemodialysis. Circulation. 2009;119(5):671-679. doi:10.1161/CIRCULATIONAHA.108.807362.

10. Mortality. https://www.usrds.org/2015/view/v2_06.aspx. Published 2016. Accessed October 15, 2016.

11. Kalantar-Zadeh K, Tortorici AR, Chen JLT, et al. Dietary Restrictions in Dialysis Patients: Is There Anything Left to Eat? Seminars in Dialysis. 2015;28(2):159-168. doi:10.1111/sdi.12348.

12. Andrew N h., Engel B, Hart K, Passey C, Beaden S. Micronutrient intake in haemodialysis patients. Journal of Human Nutrition \& Dietetics. 2008;21(4):375-376. doi:10.1111/j.1365-277X.2008.00881_4.x.

13. Meuleman Y, Brinke L, Kwakernaak A, et al. Perceived Barriers and Support Strategies for Reducing Sodium Intake in Patients with Chronic Kidney Disease: a Qualitative Study. International Journal of Behavioral Medicine. 2015;22(4):530-539. doi:10.1007/s12529-014-9447-x.

14. Hollingdale R, Sutton D, Hart K. Facilitating dietary change in renal disease: investigating patients’ perspectives. Journal of Renal Care. 2008;34(3):136-142.

15. Scribner BH. Can antihypertensive medications control BP in haemodialysis patients: yes or no? Nephrol Dial Transplant. 1999;14(11):2599-2601. doi:10.1093/ndt/14.11.2599. 
16. Kayikcioglu M, Tumuklu M, Ozkahya M, et al. The benefit of salt restriction in the treatment of end-stage renal disease by haemodialysis. Nephrol Dial Transplant. 2009;24(3):956-962. doi:10.1093/ndt/gfn599.

17. Maduell F, Navarro V. Dietary salt intake and blood pressure control in haemodialysis patients. Nephrol Dial Transplant. 2000;15(12):2063-2063. doi:10.1093/ndt/15.12.2063.

18. Rahman M, Griffin V. Patterns of antihypertensive medication use in hemodialysis patients. American Journal Of Health-System Pharmacy: AJHP: Official Journal Of The American Society Of Health-System Pharmacists. 2004;61(14):1473-1478.

19. Chazot C. Use of a Restricted-Salt Diet. Seminars in Dialysis. 2009;22(5):482-486. doi:10.1111/j.1525-139X.2009.00642.x.

20. Biruete A, Jeong JH, Barnes J, Wilund K. Modified Nutritional Recommendations to Improve Dietary Patterns and Outcomes in Hemodialysis Patients. Journal of Renal Nutrition. 2016.

21. Ozkahya M, Ok E, Cirit M, et al. Regression of left ventricular hypertrophy in haemodialysis patients by ultrafiltration and reduced salt intake without antihypertensive drugs. Nephrol Dial Transplant. 1998;13(6):1489-1493. doi:10.1093/ndt/13.6.1489.

22. Wizemann V, Wabel P, Chamney $\mathrm{P}$, et al. The mortality risk of overhydration in haemodialysis patients. Nephrol Dial Transplant. 2009;24(5):1574-1579. doi:10.1093/ndt/gfn707.

23. Leon J, Albert J, Gilchrist G, et al. Improving Albumin Levels Among Hemodialysis Patients: A Community-Based Randomized Controlled Trial. American Journal of Kidney Disease. 2006;48(1):28-36. 
24. Sullivan C, Sayre SS, Leon JB, et al. Effect of Food Additives on Hyperphosphatemia Among Patients With End-stage Renal Disease A Randomized Controlled Trial. Journal of the American Medical Association. 2009;301(6):629-635.

25. Nunes JAW, Anderson CAM, Greene JH, Ikizler TA, Cavanaugh KL. Results of a novel screening tool measuring dietary sodium knowledge in patients with chronic kidney disease. BMC Nephrology. 2015;16:42. doi:10.1186/s12882-015-0027-3.

26. Bowman J, Mogensen L, Marsland E, Lannin N. The development, content validity and inter-rater reliability of the SMART-Goal Evaluation Method: A standardised method for evaluating clinical goals. Australian Occupational Therapy Journal. 2015;62(6):420-427. doi:10.1111/1440-1630.12218.

27. Ok E, Asci G, Chazot C, Ozkahya M, Mees EJD. Controversies and problems of volume control and hypertension in haemodialysis. The Lancet. 2016;388(10041):285-293. doi:10.1016/S0140-6736(16)30389-0.

28. Charra B, Chazot C. Review Articles The Neglect of Sodium Restriction in Dialysis Patients: A Short Review. Hemodialysis International. 2003;7(4):342-347. doi:10.1046/j.1492-7535.2003.00060.x.

29. Voroneanu L, Cusai C, Hogas S, et al. The relationship between chronic volume overload and elevated blood pressure in hemodialysis patients: use of bioimpedance provides a different perspective from echocardiography and biomarker methodologies. International Urology And Nephrology. 2010;42(3):789-797. doi:10.1007/s11255-010-9767-y.

30. Baraz S, Parvardeh S, Mohammadi E, Broumand B. Dietary and fluid compliance: an educational intervention for patients having haemodialysis. Journal of Advanced Nursing. 2010;66(1):60-68. doi:10.1111/j.1365-2648.2009.05142.x. 
31. de Brito-Ashurst I, Perry L, Sanders TAB, Thomas JE, Yaqoob MM, Dobbie H. Barriers and facilitators of dietary sodium restriction amongst Bangladeshi chronic kidney disease patients. Journal of Human Nutrition \& Dietetics. 2011;24(1):86-95. doi:10.1111/j.1365277X.2010.01129.x.

32. Gutiérrez OM. Sodium and phosphorus-based food additives: persistent but surmountable hurdles in the management of nutrition in chronic kidney disease. Adv Chronic Kidney Dis. 2013;20(2):150-156. doi:10.1053/j.ackd.2012.10.008.

33. Walsh E, Lehane E. An exploration of the relationship between adherence with dietary sodium restrictions and health beliefs regarding these restrictions in irish patients receiving haemodialysis for end-stage renal disease. Journal of Clinical Nursing. 2011;20(3/4):331-340. doi:10.1111/j.1365-2702.2010.03348.x.

34. Brito-Ashurst I, Perry L, Sanders T a. B, Thomas JE, Dobbie H, Yaqoob MM. Applying research in nutrition education planning: a dietary intervention for Bangladeshi chronic kidney disease patients. Journal of Human Nutrition \& Dietetics. 2013;26(4):403-413. doi:10.1111/jhn.12022.

35. Schlatter S, Ferrans CE. Teaching program effects on high phosphorus levels in patients receiving hemodialysis. ANNA J. 1998;25(1):31-36; discussion 37-38.

36. Carrero JJ, Stenvinkel P, Cuppari L, et al. Etiology of the protein-energy wasting syndrome in chronic kidney disease: a consensus statement from the International Society of Renal Nutrition and Metabolism (ISRNM). J Ren Nutr. 2013;23(2):77-90. doi:10.1053/j.jrn.2013.01.001.

37. Sun Y, Roth DL, Ritchie CS, Burgio KL, Locher JL. Reliability and Predictive Validity of Energy Intake Measures from the 24-Hour Dietary Recalls of Homebound Older 
Adults. Journal of the American Dietetic Association. 2010;110(5):773-778.

doi:10.1016/j.jada.2010.02.003.

38. Natarajan L, Minya Pu, Juanjuan Fan, et al. Measurement Error of Dietary Self-Report in Intervention Trials. American Journal of Epidemiology. 2010;172(7):819-827. doi:10.1093/aje/kwq216.

39. Hemodialysis. The National Kidney Foundation. https://www.kidney.org/atoz/content/hemodialysis. Published January 11, 2016. Accessed October 15, 2016.

40. Peritoneal Dialysis: What You Need to Know. The National Kidney Foundation. https://www.kidney.org/atoz/content/peritoneal. Published January 7, 2016. Accessed January 7, 2018.

41. Sodium (Chloride). Linus Pauling Institute. http://lpi.oregonstate.edu/mic/minerals/sodium. Published April 23, 2014. Accessed January 25, 2017.

42. USRDS. https://www.usrds.org/2015/view/Default.aspx. Accessed January 7, 2018.

43. Sanghavi S, Whiting S, Uribarri J. Potassium Balance in Dialysis Patients. Seminars in Dialysis. 2013;26(5):597-603. doi:10.1111/sdi.12123.

44. López CM. Cultural Diversity and the Renal Diet: The Hispanic Population. Nephrology Nursing Journal. 2008;35(1):69-72.

45. Antinoro L. EN Rates 12 Common Food Additives As Safe Or Sorry Ingredients. (Cover story). Environmental Nutrition. 2008;31(5):1-4. 
46. Lynch KE, Lynch R, Curhan GC, Brunelli SM. Prescribed Dietary Phosphate Restriction and Survival among Hemodialysis Patients. Clin J Am Soc Nephrol. 2011;6(3):620-629. doi:10.2215/CJN.04620510.

47. Noori N, Sims JJ, Kopple JD, et al. Organic and Inorganic Dietary Phosphorus and Its Management in Chronic Kidney Disease. Iranian Journal of Kidney Diseases. 2010;4(2):89-100.

48. Shewfelt RL, Orta-Ramirez A, Clarke AD. Introducing Food Science. Grasas y Aceites. 2016;67(3):e154-e154.

49. Campbell N, Legowski B, Legetic B, Nilson E, L’Abbé M. Inaugural Maximum Values for Sodium in Processed Food Products in the Americas. Journal of Clinical Hypertension. 2015;17(8):611-613. doi:10.1111/jch.12553.

50. Chen J, Peng H, Zhang K, et al. The Insufficiency Intake of Dietary Micronutrients Associated with Malnutrition-Inflammation Score in Hemodialysis Population. PLoS ONE. 2013;8(6):1-6. doi:10.1371/journal.pone.0066841.

51. Onbe H, Oka M, Shimada M, Motegi E, Motoi Y, Okabe A. Defining the culture and attitude towards dietary management actions in people undergoing haemodialysis. Journal Of Renal Care. 2013;39(2):90-95. doi:10.1111/j.1755-6686.2013.12011.x.

52. Lopes AA, Bragg-Gresham JL, Ramirez SPB, et al. Prescription of antihypertensive agents to haemodialysis patients: time trends and associations with patient characteristics, country and survival in the DOPPS. Nephrol Dial Transplant. 2009;24(9):2809-2816. doi:10.1093/ndt/gfp212. 
53. Flanigan MJ. How Should Dialysis Fluid Be Individualized for the Chronic Hemodialysis Patient? Seminars in Dialysis. 2008;21(3):226-229. doi:10.1111/j.1525139X.2008.00428.x.

54. Causland FRM, Waikar SS, Brunelli SM. The relevance of dietary sodium in hemodialysis. Nephrol Dial Transplant. 2013;28(4):797-802. doi:10.1093/ndt/gfs452.

55. Asci G, Marcelli D, Celtik A, et al. Comparison of Turkish and US haemodialysis patient mortality rates: an observational cohort study. Clin Kidney J. 2016;9(3):476-480. doi: $10.1093 / \mathrm{ckj} / \mathrm{sfw} 027$.

56. Hendriksen M a H, Verkaik-Kloosterman J, Noort MW, van Raaij JMA. Nutritional impact of sodium reduction strategies on sodium intake from processed foods. European Journal of Clinical Nutrition. 2015;69(7):805-810. doi:10.1038/ejen.2015.15.

57. Rodrigues-Telini LS, Beduschi G de C, Caramori JCT, Castro JH, Martin LC, Barretti P. Effect of dietary sodium restriction on body water, blood pressure, and inflammation in hemodialysis patients: a prospective randomized controlled study. Int Urol Nephrol. 2013;46(1):91-97. doi:10.1007/s11255-013-0382-6.

58. Agarwal R, Nissenson AR, Batlle D, Coyne DW, Trout JR, Warnock DG. Prevalence, treatment, and control of hypertension in chronic hemodialysis patients in the United States. The American Journal of Medicine. 2003;115(4):291-297. doi:10.1016/S00029343(03)00366-8. 
Table 1. Baseline Patient Characteristics

\begin{tabular}{|lc|}
\hline Demographics and Clinical Data & Participants (n=23) \\
Age in years (standard deviation) & $55.7(13.3)$ \\
Male (\%) & $12(52.2)$ \\
Hispanic (\%) & $1(4.5)$ \\
African American (\%) & $9(40.9)$ \\
Patients w/ Diabetes (\%) & $10(45.5)$ \\
Avg. Weight in kg (standard deviation) & $93.6( \pm 24.4)$ \\
Avg. IDWG in kg (standard deviation) & $3.15( \pm 1.1)$ \\
\hline
\end{tabular}


Table 2. Body Fluid Levels

\begin{tabular}{|lccc|}
\hline Bioimpedance & Pre-Intervention & Post-Intervention & p-value \\
Spectroscopy Data & & & \\
Total Body Water in Liters & $45.8( \pm 10.8)$ & $44.3( \pm 8.8)$ & 0.10 \\
Total Body Water \% & $49.8( \pm 7.1)$ & $49.4( \pm 6.4)$ & 0.61 \\
\hline
\end{tabular}

All values presented are mean (standard deviation). $n=23$ 
Table 3. Blood Pressure and Medication Outcomes

\begin{tabular}{|lccc|}
\hline Blood Pressure Data & Pre-Intervention & Post-Intervention & p-value \\
Systolic BP in mmHg & $160( \pm 25)$ & $156( \pm 23)$ & 0.56 \\
Diastolic BP in mmHg & $81( \pm 20)$ & $79( \pm 15)$ & 0.73 \\
\# BP Medications & $3( \pm 1)$ & $2( \pm 1)$ & 0.003 \\
\end{tabular}

All values presented are mean (standard deviation). $n=23$ 
Table 4. Dietary Intake Evaluation

\begin{tabular}{|lccc|}
\hline Dietary Intake Data & Pre-Intervention & Post-Intervention & p-value \\
Energy Intake in kcals & $1588( \pm 1061)$ & $1366( \pm 455)$ & 0.38 \\
Protein Intake in grams & $61( \pm 30)$ & $56( \pm 21)$ & 0.60 \\
Protein Intake in g/kcal & $0.72( \pm 0.50)$ & $0.65( \pm 0.28)$ & 0.79 \\
Sodium Intake in mg & $2886( \pm 1570)$ & $2315( \pm 1095)$ & 0.13 \\
Sodium Intake in mg/kcal & $1.96( \pm 0.84)$ & $1.69( \pm 0.53)$ & 0.16 \\
\hline
\end{tabular}

All values presented are mean (standard deviation). $n=23$ 
Table 5. Dietary Knowledge Evaluation

\begin{tabular}{|lccr|}
\hline Dietary Knowledge & Pre-Intervention & Post-Intervention & p-value \\
Protein Knowledge \% Correct & $81( \pm 18)$ & $84( \pm 14)$ & 0.25 \\
Phosphorus Knowledge \% Correct & $66( \pm 15)$ & $74( \pm 14)$ & 0.04 \\
Sodium Knowledge \% Correct & $68( \pm 27)$ & $76( \pm 30)$ & 0.06 \\
\hline
\end{tabular}

All values presented are mean (standard deviation). $n=23$ 
Figure 1. Reduction in Liters of Volume Overload Following Intervention.

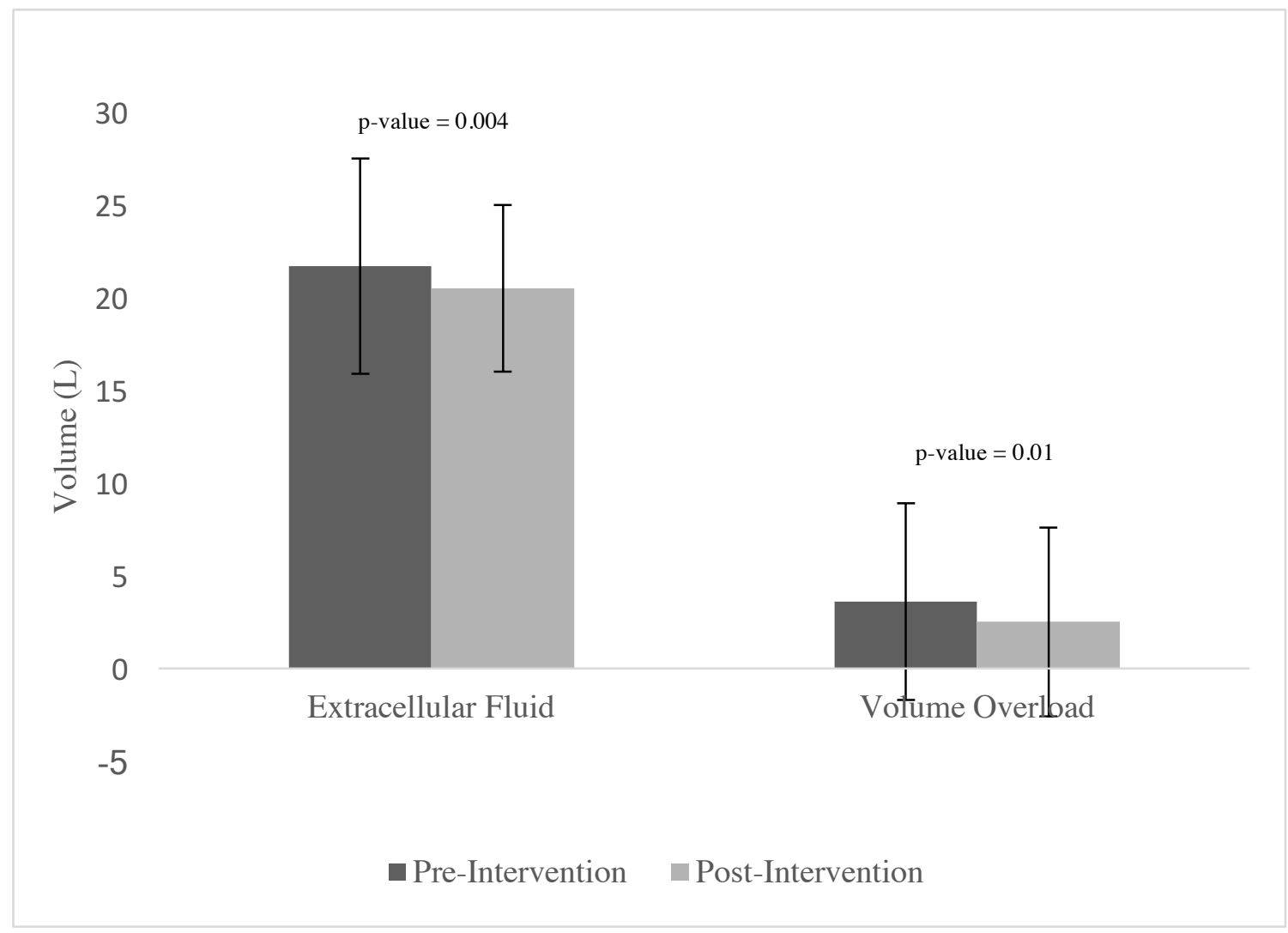

All values presented are mean $( \pm$ standard deviation $)$ 
Figure 2. Significant Post-Intervention Improvement in Volume Overload Percentage.

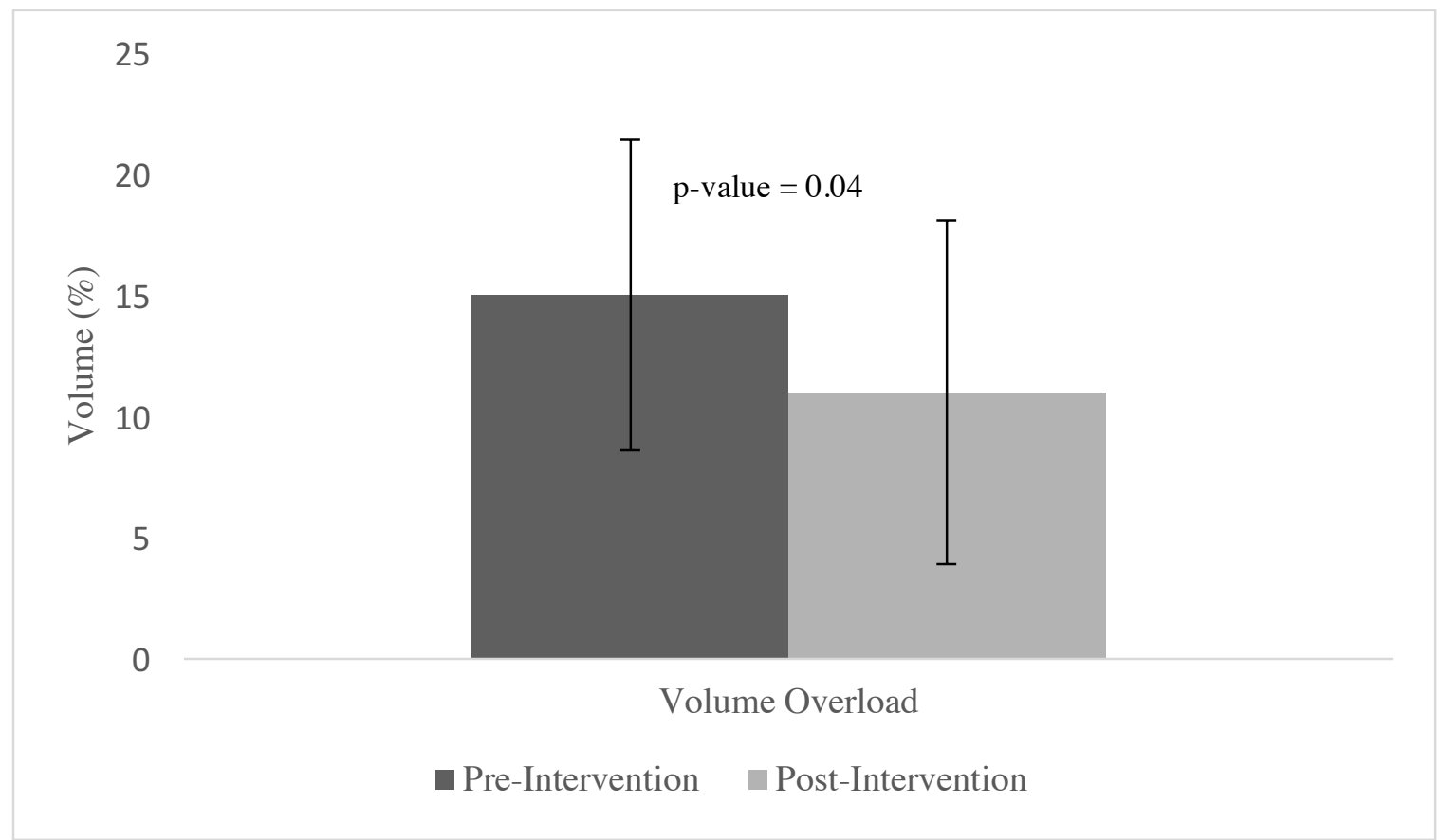

All values presented are mean $( \pm$ standard deviation $)$ 
APPENDIX A: 24-HOUR RECALL FORM

Pt ID:

Researcher:

Date Consumed:

Date Recorded:

\begin{tabular}{|c|c|c|c|c|}
\hline Meal & Time & Food/Drink & Portion/Brand & Location \\
\hline & & & & \\
\hline & & & & \\
\hline & & & & \\
\hline & & & & \\
\hline & & & & \\
\hline & & & & \\
\hline & & & & \\
\hline & & & & \\
\hline & & & & \\
\hline & & & & \\
\hline & & & & \\
\hline & & & & \\
\hline & & & & \\
\hline & & & & \\
\hline & & & & \\
\hline & & & & \\
\hline & & & & \\
\hline & & & & \\
\hline & & & & \\
\hline & & & & \\
\hline & & & & \\
\hline & & & & \\
\hline & & & & \\
\hline & & & & \\
\hline & & & & \\
\hline & & & & \\
\hline & & & & \\
\hline & & & & \\
\hline & & & & \\
\hline & & & & \\
\hline & & & & \\
\hline & & & & \\
\hline & & & & \\
\hline & & & & \\
\hline & & & & \\
\hline & & & & \\
\hline
\end{tabular}


Pt ID:

Goal Setting:

\begin{tabular}{|c|c|c|}
\hline Initial Date & Goal (SMART) & Aim Achievement Date \\
\hline Date: _L_ I & & Date: ___ I _ \\
\hline Date: _L_ I & & Date: ___ I \\
\hline Date: _L I I & & Date: $\quad 1$ \\
\hline Date: _L_ I & & Date: \\
\hline
\end{tabular}

\section{Current Habits:}

\begin{tabular}{|l|l|}
\hline Positive Habit & Strategies for Maintaining Habit \\
\hline & \\
\hline & \\
\hline Concerning Habit & \\
\hline & Strategies for Improving Habit \\
\hline & \\
\hline & \\
\hline & \\
\hline
\end{tabular}


Pt ID:

Researcher:

\section{Current Goal \#1}

\begin{tabular}{|c|c|}
\hline Initial Set Date: & Actual Achievement Date: \\
\hline Aim Achievement Date: & $\begin{aligned} & \text { Are all SMART components met? (yes/no) } \\
& \text { - } \text { Specific: } \\
& \text { - } \text { Measurable: } \\
& \text { - } \text { Attainable: } \\
& \text { - } \text { Relevant: } \\
& \text { - } \text { Time Related: }\end{aligned}$ \\
\hline \multicolumn{2}{|c|}{ Did the patient achieve this goal? How did they achieve it, or why did they not achieve it? } \\
\hline \multicolumn{2}{|c|}{$\begin{array}{l}\text { If the goal was not achieved, restructure the goal to the SMART model and patient } \\
\text { needs: } \\
\text { - Was it specific enough to achieve? } \\
\text { - Can you specify the measurement to be numerical/unit based rather than more/less? } \\
\text { - } \quad \text { Can this patient achieve this action within the specific time period? } \\
\text { - Is there a clear time frame? } \\
\text { - Utilize the goal builder section to improve this goal for better achievement }\end{array}$} \\
\hline \multicolumn{2}{|c|}{$\begin{array}{l}\text { If the goal was achieved: } \\
\text { - Use the goal builder section to develop this goal even further for the patient } \\
\text { - } \quad \text { Use the goal builder section to create a new goal in an area that has not yet been } \\
\text { approached by this patient } \\
\text { - } \quad \text { Use successful approaches of the last goal to fuel the new goal }\end{array}$} \\
\hline
\end{tabular}

\section{Goal Builder \#1:}

\begin{tabular}{|c|c|}
\hline Current Date: ___ I _ I & Aim Achievement Date: \\
\hline Goal: & $\begin{aligned} & \text { Are all SMART components met? (yes/no) } \\
& \text { - } \text { Specific: } \\
& \text { - } \text { Measurable: } \\
& \text { - } \text { Attainable: } \\
& \text { - } \text { Relevant: } \\
& \text { - } \text { Time Related: }\end{aligned}$ \\
\hline \multicolumn{2}{|c|}{ Additional notes or sub-goals for success: } \\
\hline
\end{tabular}

